\title{
Indicadores do processo de ensino aprendizagem do registro em prontuário no contexto da Terapia Ocupacional hospitalar
}

\author{
Tatiana Barbieri Bombarda \\ Doutora em Terapia Ocupacional pelo Programa de Pós-Graduação em Terapia Ocupacional da Universidade \\ Federal de São Carlos - UFSCar. Professora Adjunta no Departamento de Terapia Ocupacional da UFSCar. \\ $\triangle$ tatibb_to@yahoo.com.br
}

\begin{abstract}
Regina Helena Vitale Torkomian Joaquim
Doutora em Educação Especial pelo Programa de Educação Especial da Universidade Federal de São Carlos - UFSCar. Pós-doutorado pelo Departamento de Saúde Materno-Infantil e Saúde Pública da Escola de Enfermagem - USP/RP. Professora Associada do Departamento de Terapia Ocupacional da UFSCar.
\end{abstract}

\begin{abstract}
Resumo:
Indicadores sobre inconformidades na prática dos registros em prontuário realizados por terapeutas ocupacionais são evidenciados na literatura, sendo questionado o preparo ofertado no âmbito da graduação e pós-graduação sobre a formação dada a este procedimento. Este estudo visa compreender o processo de ensino aprendizagem do registro em prontuário na área de contextos hospitalares presentes nos cursos brasileiros de graduação em Terapia Ocupacional. Trata-se de uma pesquisa de abordagem quantitativa, sendo os participantes docentes de disciplinas teóricas e de estágio profissionalizante em contextos hospitalares dos cursos de Terapia Ocupacional, bem como alunos em prática de estágio profissionalizante em Terapia Ocupacional hospitalar. Utilizou-se como instrumento de coleta de dados um questionário online, sendo os dados analisados por meio de estatística descritiva e teste Qui-Quadrado. Participaram do estudo 15 docentes e 33 alunos, os quais sinalizaram a narrativa livre como principal modelo de registro utilizado nas anotações em prontuário. Foram constatadas fragilidades no conteúdo de registros de avaliação, intervenção e alta, e, escasso ensino sobre a elaboração de indicadores de produtividade, qualidade assistencial e compra e controle de materiais. As inconsistências verificadas no processo de ensino aprendizagem do registro em prontuário no âmbito da graduação brasileira indicam a necessidade de maiores investimentos na formação sobre a prática documental para assim garantir anotações qualificadas na prática profissional.
\end{abstract}

Palavras-chave: Registros, ensino, terapia ocupacional, hospitais.

\section{Indicators of the teaching-learning process of medical record registration in the context of hospital Occupational Therapy}

\begin{abstract}
:
Indicators of weaknesses in the practice of medical records made by occupational therapists are evidenced in the literature, being the preparation offered, under the undergraduate and postgraduate training on this procedure, what is being questioned. This study seek to understand the process of teaching learning about medical records in the area of hospital contexts present in brazilians undergraduate courses in Occupational Therapy. This is a research with a quantitative approach. Participants were lecturers responsible for theoretical and professional internship disciplines in hospital contexts of active Occupational Therapy courses in Brazil, as well as students in professional internship practice in Occupational Therapy in hospital contexts. An online questionnaire was used as a data collection instrument, and the data were analyzed by descriptive statistics e chi square test. Fifteen teachers and 33 students participated in the study, who signaled the free narrative as the main
\end{abstract}


registration model used in the medical records. Non-conformities were found in the content of assessment, intervention and discharge records, as well as the scarce offer of teaching on the elaboration of productivity indicators, quality of care and purchase and control of materials. The inconsistencies found in the teaching and learning process of medical record registration in the Brazilian undergraduate level indicate the need for greater investments in formation on documentary practice in order to ensure qualified records in professional practice.

Keywords: Records, teaching, occupational therapy, hospitals.

\title{
Indicadores del proceso de enseñanza y aprendizaje de los registros clínicos en el contexto de la Terapia Ocupacional hospitalaria
}

\begin{abstract}
Resumen:
Los indicadores sobre las inconformidades en la práctica de los registros realizados por terapeutas ocupacionales se evidencian en la literatura, y se cuestiona la preparación ofrecida en los cursos de pregrado y posgrado sobre la capacitación impartida a este procedimiento. El objetivo de este estudio es comprender el proceso de enseñanza aprendizaje de los registros clínicos en el área hospitalaria presentes en cursos de pregrado brasileños de terapia ocupacional. Esta es una investigación con un enfoque cuantitativo, siendo los participantes, profesores de disciplinas teóricas y pasantías profesionales en los contextos hospitalarios de cursos de Terapia Ocupacional, así como estudiantes en prácticas profesionales en Terapia Ocupacional hospitalaria. Se utilizó un cuestionario en línea como instrumento de recopilación de datos, y los datos se analizaron mediante estadística descriptiva y la prueba de Chi-cuadrado. Quince profesores y 33 estudiantes participaron en el estudio, que señalaron la narrativa libre como el principal modelo de registro utilizado en las notas clínicas. Se encontraron debilidades en el contenido de la evaluación, intervención y registros de alta hospitalaria, así como poca enseñanza sobre el desarrollo de indicadores de productividad, calidad de la atención, compra y control de materiales. Las inconsistencias verificadas en el proceso de enseñanza- aprendizaje de los registros clínicos dentro del alcance del curso de pregrado brasileño indican la necesidad de mayores inversiones en la formación sobre la práctica del registro documental para garantizar anotaciones y evoluciones calificadas en la práctica profesional.

Palabras clave: Registros, enseñanza, terapia ocupacional, hospitales.
\end{abstract}

\section{INTRODUÇÃO}

A Terapia Ocupacional em contextos hospitalares apresenta-se no Brasil como um campo profissional em expansão. Esta afirmação respalda-se em indicadores como os de que aproximadamente $40 \%$ dos terapeutas ocupacionais que compõe a categoria profissional atuam em hospitais (PINHEIRO, 2014), sendo as instituições hospitalares (secundárias, terciárias, hospitais gerais e especializados) um mercado de trabalho crescente (DE CARLO, 2014).

Esta ampliação da atuação do terapeuta ocupacional no âmbito hospitalar brasileiro ocorre atrelada ao crescimento de atividades de ensino, de pesquisa, de fundamentação técnica científica, de regulamentações da prática (portarias, resoluções), de grupos de 
pesquisa e de eventos científicos com a especificidade de temas relativos ao campo (DE CARLO, 2014).

No ano de 2018, o exame para a concessão do registro do título de especialista na área de Terapia Ocupacional abrangeu seis especialidades, a saber: Terapia Ocupacional em Acunpuntura, Terapia Ocupacional em Contextos Sociais, Terapia Ocupacional em Contextos Hospitalares, Terapia Ocupacional em Gerontologia, Terapia Ocupacional em Saúde da Família e Terapia Ocupacional em Saúde Mental (COFFITO, 2018), sendo a especialidade de contextos hospitalares a área com maior número de inscritos (COFFITO, 2018b).

De acordo com o art. 6º da resolução 429/2013 do Conselho Federal de Fisioterapia e Terapia Ocupacional - COFFITO, que disciplina a especialidade de Terapia Ocupacional em Contextos Hospitalares, os conhecimentos mínimos para atuação do terapeuta ocupacional no hospital perpassam por múltiplos aspectos que envolvem desde normas de biossegurança, controle de infecção hospitalar e bioética, como conteúdos de tanatologia, farmacologia, suporte básico da vida, fundamentos teórico metodológicos da profissão, entre outros (COFFITO, 2013), fator que denota um amplo repertório de conhecimentos técnicos que demanda atenção ao processo de formação nesta área.

Segundo Fangel et al. (2018), é importante considerar para a formação no contexto hospitalar a integração de aspectos voltados a formação teórica, inserção na prática, articulação com a equipe, manejo com o paciente e registro do que foi realizado.

Em específico sobre os registros, a World Federation of Occupational Therapists (WFOT), organização internacional voltada à promoção e desenvolvimento da Terapia Ocupacional, recomenda dentre os padrões mínimos para educação de terapeutas ocupacionais o desenvolvimento da habilidade de execução de registros de qualidade, pautados em preceitos éticos e legislativos (WFOT, 2016).

Todavia, diversos estudos têm apontado indicadores sobre inconformidades na prática dos registros em prontuário realizados por terapeutas ocupacionais, sendo questionado o preparo ofertado no âmbito da graduação e pós-graduação sobre a formação dada a este procedimento (PANZERI, 2013; PELISSARI; PALHARES, 2015; BOMBARDA; PALHARES, 2015; BUCHANAN JELSMA; SIEGRIED, 2016). 
Para Fearing (1993), o aprendizado sobre a documentação em Terapia Ocupacional é um processo desorganizado e insatisfatório, o que denota a importância de investigações voltadas à melhor compreensão do processo educacional sobre o registro em prontuário.

Diante do exposto, este estudo visou compreender o processo de ensino aprendizagem do registro em prontuário na área de contextos hospitalares presentes nos cursos brasileiros de graduação em Terapia Ocupacional.

\section{METODOLOGIA}

Trata-se de um estudo transversal, de abordagem quantitativa.

De acordo com Gil (2002), as pesquisas com abordagem quantitativa pautam-se na organização dos dados em tabelas por meio de tratamento estatístico, fator que facilita a ordenação lógica do trabalho, permitindo a descrição das informações.

Os participantes foram docentes responsáveis por disciplinas teóricas e de estágio profissionalizante em contextos hospitalares dos cursos de Terapia Ocupacional ativos do Brasil, bem como os alunos em prática de estágio profissionalizante em Terapia Ocupacional nos contextos hospitalares.

Foram excluídos docentes responsáveis por disciplinas teórico práticas e os que apontaram não estar mais como responsáveis pelas disciplinas teóricas ou de estágio profissionalizante no contexto hospitalar. Também foram excluídos os alunos que manifestaram estar em estágio profissionalizante referente a outra especialidade, mas fazendo uso do hospital enquanto equipamento de atuação.

Os instrumentos de coleta de dados foram desenvolvidos pela pesquisadora sendo um questionário voltado aos docentes e outro aos alunos em prática de estágio profissionalizante. Os questionários foram fundamentados nas diretrizes nacionais e internacionais da profissão acerca do registro em prontuário, em levantamento bibliográfico sobre o tema e em experiências profissionais da pesquisadora. Os instrumentos foram avaliados por juízes (profissionais experientes na área) e sistematizados em plataforma online. 
A estruturação de ambos os questionários consistiu em 4 partes, sendo neste artigo apresentadas as informações concernentes a caracterização dos participantes e compreensão acerca do processo de ensino aprendizagem do procedimento do registro em prontuário informadas por meio de questões de múltiplas escolhas.

O contato com os participantes foi realizado por e-mail, via coordenação de curso, no primeiro semestre de 2018, sendo encaminhada uma mensagem convite que explicitava os objetivos do estudo, o parecer de aprovação do comitê de ética em pesquisa ( $n^{\circ}$ 1.894.915) e o link de acesso ao questionário online.

Os dados obtidos foram analisados por estatística descritiva, a qual consiste em um resumo das principais características de um conjunto de dados, por meio de tabelas, gráficos e índices numéricos (GUIMARÃES, 2008). Em complemento, houve a aplicação do teste QuiQuadrado através do software IBM SPSS Statistics versão 22, em que para se afirmar a ocorrência de associações, considera-se que as relações entre as variáveis apresentem o valor designado por "p" menor que 0,05 $(p<0,05)$.

Como referencial analítico foram utilizadas as diretrizes nacionais e internacionais da profissão sobre registros e documentação, tais como: a resolução COFFITO-415/2012, Guidelines for Documentation of Occupational Therapy (AOTA), Practice Guideline: client records e Practice Standards for Managing Client Information.

\section{RESULTADOS}

Por meio de contato com as coordenações de curso foram identificados 32 docentes, contudo $15(46,8 \%)$ deles realizaram participação na pesquisa.

A caracterização dos docentes abrangeu totalidade do sexo feminino, com média de idade de 45 anos (sendo a menor idade 29 e a maior 61) e tempo médio de formação de 22 anos (variável de 7 à 41 anos). A média do tempo de experiência na docência consistiu em 11 anos (variando entre 10 meses e 40 anos) e a média do tempo de experiência na área hospitalar (considerando docência e prática profissional) foi equivalente a 10 anos (sendo mínimo 3 meses e máximo de 38 anos). 
Em relação à amostra de alunos, foi obtida participação de 33 estagiários de Terapia Ocupacional em contextos hospitalares, sendo o perfil apresentado de predominância feminina (94\%), média de idade de 28 anos ( $d p=10)$, sendo o mais novo com 21 anos e o mais velho com 54 anos.

Verificou-se que 23 alunos (69,7\%) relataram ter tido disciplina de contextos hospitalares e destes $6(26,1 \%)$ afirmaram que na disciplina não foram ofertados conteúdos sobre a prática de registro em prontuário.

Salienta-se que 26 alunos participantes $(78,8 \%)$ já haviam efetivado estágios prévios em outras áreas e que no estágio atual de contextos hospitalares 7 deles $(21,2 \%)$ indicaram ter acesso ao prontuário apenas para leitura.

Tabela 1- Frequências ordenadas em ordem decrescente das orientações feitas pelos docentes em relação ao registro de avaliação, intervenção e alta.

\begin{tabular}{|c|c|c|}
\hline INFORMAÇÕES OBRIGATÓRIAS EXIGIDAS PELOS DOCENTES NOS REGISTROS DE AVALIAÇÃO: & $\mathrm{n}$ & $\%$ \\
\hline Quem foi o informante (paciente, acompanhante) & 15 & 100,0 \\
\hline $\begin{array}{l}\text { Informações sobre o modo como o paciente se apresentou } \\
\text { (orientação tempo-espacial, humor, acompanhado, etc) }\end{array}$ & 15 & 100,0 \\
\hline Informações sobre a queixa do paciente & 15 & 100,0 \\
\hline $\begin{array}{l}\text { Informações sobre instrumentos utilizados na avaliaçã̃o de } \\
\text { componentes motores, cognitivos, sociais e espirituais }\end{array}$ & 15 & 100,0 \\
\hline data & 15 & 100,0 \\
\hline Assinatura do aluno & 15 & 100,0 \\
\hline Informações sobre dados pessoais do paciente & 14 & 93,3 \\
\hline Informações acerca do perfil ocupacional e seu desempenho & 14 & 93,3 \\
\hline $\begin{array}{l}\text { Informações sobre a compreensão e aceitação do paciente em } \\
\text { relação ao processo de adoecimento e internação }\end{array}$ & 14 & 93,3 \\
\hline Informações sobre os prejuízos funcionais & 14 & 93,3 \\
\hline Aspectos relativos à família e ao cuidador & 14 & 93,3 \\
\hline Plano terapêutico & 14 & 93,3 \\
\hline Assinatura do preceptor/supervisor & 14 & 93,3 \\
\hline $\begin{array}{l}\text { Modo pelo qual o paciente chegou até o profissional } \\
\text { (busca ativa, interconsulta, demanda espontânea) }\end{array}$ & 12 & 80,0 \\
\hline Diagnóstico terapêutico ocupacional & 12 & 80,0 \\
\hline Carimbo ou registro profissional do preceptor/supervisor & 12 & 80,0 \\
\hline Carimbo ou número do RA (registro acadêmico) do estagiário & 6 & 40,0 \\
\hline Informações sobre o prognóstico TO & 5 & 33,3 \\
\hline Quantitativo estimado de atendimentos & 3 & 20,0 \\
\hline Outras* & 1 & 6,7 \\
\hline
\end{tabular}




\begin{tabular}{|c|c|c|}
\hline INFORMAÇÕES OBRIGATÓRIAS EXIGIDAS PELOS DOCENTES NOS REGISTROS DE INTERVENÇÃO & $\mathrm{n}$ & $\%$ \\
\hline Objetivo do atendimento & 15 & 100,0 \\
\hline Informações sobre desempenho do paciente na intervenção proposta & 15 & 100,0 \\
\hline Informações sobre o que foi alcançado & 15 & 100,0 \\
\hline Informações acerca de quais orientações foram realizadas & 15 & 100,0 \\
\hline Local onde foi realizado o atendimento & 14 & 93,3 \\
\hline $\begin{array}{l}\text { Informações sobre o modo como o paciente se apresenta } \\
\text { (orientação tempo-espacial, humor, acompanhado, etc) }\end{array}$ & 14 & 93,3 \\
\hline Abordagem e recursos empregados & 14 & 93,3 \\
\hline Data & 14 & 93,3 \\
\hline Assinatura do aluno & 14 & 93,3 \\
\hline Assinatura do preceptor/supervisor & 13 & 86,7 \\
\hline Carimbo ou número do registro profissional do preceptor/supervisor & 13 & 86,7 \\
\hline Informações sobre pessoas presentes no atendimento e participações & 11 & 73,3 \\
\hline $\begin{array}{l}\text { Informações acerca de discussões interdisciplinares, contatos telefônicos, } \\
\text { acompanhamento de procedimentos, entre outros }\end{array}$ & 11 & 73,3 \\
\hline Número do RA (registro acadêmico) do aluno estagiário & 6 & 40,0 \\
\hline Outros** & 1 & 6,7 \\
\hline INFORMAÇÕES OBRIGATÓRIAS EXIGIDAS PELOS DOCENTES NOS REGISTROS DE ALTA: & $\mathrm{n}$ & $\%$ \\
\hline Resumo do que foi trabalhado e alcançado & 14 & 100,0 \\
\hline Informações sobre encaminhamentos para seguimento no pós-alta & 14 & 100,0 \\
\hline Informações acerca de quais orientações foram realizadas & 14 & 100,0 \\
\hline Data & 14 & 100,0 \\
\hline Assinatura do aluno & 13 & 86,7 \\
\hline Assinatura do preceptor/supervisor & 13 & 86,7 \\
\hline Carimbo ou número do registro profissional do preceptor/supervisor & 12 & 80,0 \\
\hline $\begin{array}{l}\text { Informações sobre o modo como o paciente se apresenta } \\
\text { (orientação tempo-espacial, humor, acompanhado, etc) }\end{array}$ & 12 & 80,0 \\
\hline Informações sobre quem estava presente na orientação de alta & 9 & 60 \\
\hline Número do RA (registro acadêmico) do aluno estagiário & 6 & 40 \\
\hline
\end{tabular}

* Foi sinalizado na opção outros a orientação por um docente em relação a explicitação da conduta terapêutica para o próximo atendimento no registro de avaliação.

** Foi sinalizado a exigência por um docente da inserção do $n^{\circ}$ do $R G$ do aluno junto a sua assinatura.

Fonte: elaborado pelos próprios autores.

De acordo com as frequências absolutas (n) e relativas (\%) foi possível perceber que há inconsistências no processo de ensino do registro em prontuário. Considerando os índices abaixo dos 70\%, como principais inconformidades, observou-se nos registros de avaliação, menor exigência pelos docentes em relação ao carimbo ou número do registro do estagiário, informações sobre o prognóstico terapêutico ocupacional e acerca do quantitativo estimado de atendimentos. Já, em relação aos registros de intervenções, o item menos exigido refere- 
se ao registro acadêmico do estagiário (RA), enquanto que no registro de alta a menor exigência está atrelada às informações sobre quem estava presente na orientação de alta e acerca da identificação do número do registro acadêmico do aluno (RA).

Em relação à prática de registro, efetivada pelos alunos, também foram observadas inconsistências de conteúdos preconizados, conforme detalhado na tabela 2.

Tabela 2- Frequências ordenadas em ordem decrescente da indicação dos alunos em relação ao conteúdo incorporado nos registros de avaliação, intervenção e alta.

\begin{tabular}{|c|c|c|}
\hline $\begin{array}{l}\text { INFORMAÇÕES SINALIZADAS PELOS ALUNOS COMO CONTEÚDOS OBRIGATÓRIOS EM SEU } \\
\text { REGISTRO DE AVALIAÇÃO: }\end{array}$ & $n$ & $\%$ \\
\hline Informações sobre a queixa do paciente & 31 & 96,9 \\
\hline Informações sobre os prejuízos funcionais & 30 & 93,8 \\
\hline Data & 30 & 93,8 \\
\hline Informações acerca do perfil ocupacional e seu desempenho & 29 & 90,6 \\
\hline Quem foi o informante (paciente, acompanhante) & 28 & 87,5 \\
\hline $\begin{array}{l}\text { Informações sobre o modo como o paciente se apresentou } \\
\text { (orientação tempo-espacial, humor, acompanhado, etc) }\end{array}$ & 28 & 87,5 \\
\hline Assinatura do preceptor/supervisor & 28 & 87,5 \\
\hline Informações sobre dados pessoais do paciente & 27 & 84,4 \\
\hline Carimbo ou registro profissional do preceptor/supervisor & 27 & 84,4 \\
\hline Assinatura do aluno & 25 & 78,1 \\
\hline $\begin{array}{l}\text { Informações sobre instrumentos utilizados na avaliação de componentes motores, } \\
\text { cognitivos, sociais e espirituais }\end{array}$ & 24 & 75 \\
\hline $\begin{array}{l}\text { Informações sobre a compreensão e aceitação do paciente em relação ao processo } \\
\text { de adoecimento e internação }\end{array}$ & 22 & 68,8 \\
\hline Aspectos relativos à família e ao cuidador & 22 & 68,8 \\
\hline Diagnóstico terapêutico ocupacional & 21 & 65,6 \\
\hline $\begin{array}{l}\text { Local onde foi feita a avaliação (enfermaria, ambulatório, leito, sala de reabilitação, } \\
\text { brinquedoteca, etc) }\end{array}$ & 21 & 65,6 \\
\hline $\begin{array}{l}\text { Modo pelo qual o paciente chegou até o profissional (busca ativa, interconsulta, } \\
\text { demanda espontânea) }\end{array}$ & 19 & 59,4 \\
\hline Informações sobre o prognóstico TO & 17 & 53,1 \\
\hline Carimbo ou número do RA (registro acadêmico) do estagiário & 16 & 50 \\
\hline Plano terapêutico & 14 & 43,8 \\
\hline Quantitativo estimado de atendimentos & 5 & 15,6 \\
\hline $\begin{array}{l}\text { INFORMAÇÕES SINALIZADAS PELOS ALUNOS COMO CONTEÚDOS OBRIGATÓRIOS EM SEU } \\
\text { REGISTRO DE INTERVENÇÃO: }\end{array}$ & $n$ & $\%$ \\
\hline Informações sobre desempenho do paciente na intervenção proposta & 31 & 96,9 \\
\hline Objetivo do atendimento & 30 & 93,8 \\
\hline Data & 29 & 90,6 \\
\hline Informações acerca de quais orientações foram realizadas & 28 & 87,5 \\
\hline Assinatura do preceptor/supervisor & 28 & 87,5 \\
\hline Informações sobre o modo como o paciente se apresenta & 27 & 84,4 \\
\hline
\end{tabular}


(orientação tempo-espacial, humor, acompanhado, etc)

Carimbo ou número do registro profissional do preceptor/supervisor

Informações sobre pessoas presentes no atendimento e participações

Abordagem e recursos empregados

Informações sobre o que foi alcançado

Local onde foi realizado o atendimento

Assinatura do aluno

Número do RA (registro acadêmico) do aluno estagiário

Informações acerca de discussões interdisciplinares, contatos telefônicos,

acompanhamento de procedimentos, entre outros

\begin{tabular}{lcc}
\hline INFORMAÇõES SINALIZADAS PELOS ALUNOS COM CONTEÚDOS OBRIGATÓRIOS DE SEU & & $\%$ \\
\hline REGISTRO DE ALTA: & 29 & 93,5 \\
\hline Informações acerca de quais orientações foram realizadas & 28 & 90,3 \\
Data & 27 & 87,1 \\
Informações sobre encaminhamentos para seguimento no pós-alta & 27 & 87,1 \\
Assinatura do preceptor/supervisor & 26 & 83,9 \\
Carimbo ou número do registro profissional do preceptor/supervisor & 21 & 67,7 \\
Informações sobre o modo como o paciente se apresenta & & \\
(orientação tempo-espacial, humor, acompanhado, etc) & 21 & 67,7 \\
Assinatura do aluno & 19 & 61,3 \\
Resumo do que foi trabalhado e alcançado & 19 & 61,3 \\
Informações sobre quem estava presente na orientação de alta & 14 & 45,2
\end{tabular}

Fonte: elaborado pelos próprios autores

Considerando índices abaixo dos 70\%, em relação ao conteúdo dos registros, referente ao processo de avaliação, realizados pelos alunos, verificou-se inconsistências relacionadas às informações sobre a compreensão e aceitação do paciente em relação ao processo de adoecimento e internação, aspectos relativos à familia/cuidador, ao diagnóstico terapêutico ocupacional, acerca do local onde foi efetivada a avaliação e o modo pelo qual o paciente chegou até o profissional. Destaca-se que, aproximadamente, metade dos alunos (53\%) mencionou sobre a incorporação do prognóstico terapêutico ocupacional. Informações como o plano terapêutico e, em especial, estimativa do quantitativo de atendimentos a ser realizado emergiram como maiores déficits de conteúdo dos registros avaliativos.

Em relação aos registros das intervenções, como principais déficits foi verificada a ausência do RA do aluno estagiário e de informações sobre discussões interdisciplinares, contatos telefônicos, acompanhamento de procedimentos, entre outros. Já para os registros de alta $61,3 \%$ dos alunos (n=19) referiram inserir em suas anotações um resumo do que foi 
trabalhado e alcançado e registrar a identificação de quem recebeu as orientações de alta. É importante relatar que um aluno referiu que na maior parte das vezes, o terapeuta ocupacional não toma conhecimento da alta hospitalar, o que dificulta registros referentes a esta etapa.

Considerando que a resolução 415, do COFFITO, constitui-se como o principal documento nacional voltado a prática do registro em prontuário na área de Terapia Ocupacional, entre as questões do questionário havia uma pergunta voltada a identificação do conhecimento dos participantes sobre esta resolução. Como resultados verificou-se um elevado indíce de desconhecimento por parte dos alunos $(72,7 \%)$ e por dois dos docentes.

Como um dos componentes sinalizados como obrigatório na resolução 415 do COFFITO, está o diagnóstico terapêutico ocupacional. Em relação a este aspecto, aproximadamente metade dos docentes $(\mathrm{n}=8)$ afirmou orientar seus alunos a realizar o diagnóstico terapêutico ocupacional, a partir das informações constadas no processo de avaliação. Outros docentes referiram orientar os alunos a utilizar como diagnóstico a Classificação Internacional de Funcionalidade $(n=4)$ e o diagnóstico situacional, elemento referido pelo Metódo de Terapia Ocupacional Dinâmica (n=3).

Já os alunos afirmaram, de modo mais frequente, realizar o diagnóstico terapêutico ocupacional $(n=10)$ e diagnóstico situacional $(n=7)$. Houve também apontamentos da não efetivação do registro do diagnóstico pela dificuldade em nomeá-lo (n=6). Em complemento, houve a sinalização de alunos que realizam o diagnóstico no processo de avaliação, contudo não o registram $(n=5)$.

Destaca-se ainda que no grupo de alunos foi relatado também a aplicação de classificações como a do CID-10 e DSM-V como diagnóstico terapêutico ocupacional (n=3) e referente ao uso da Classificação Internacional de Funcionalidade - CIF ( $n=1)$.

Verificou-se que o principal formato de registro ensinado por docentes é o da narrativa livre, seguido de forma menos frequente pelo uso de roteiro pré-estruturado ou embasado na Classificação Internacional de Funcionalidade - CIF. Já os alunos, considerando a soma da indicação sobre o uso de roteiros, referiram como principal formato utilizado na prática de estágio o uso de roteiros pré-estruturados (sejam estes definidos pela instituição ou pelo setor da TO) e a narrativa livre, conforme apresentado na tabela 3. 
Tabela 3 - Informações sobre o formato de registro utilizado por docentes e alunos no processo de ensino aprendizagem.

\begin{tabular}{lccc}
\hline PRINCIPAL FORMATO DO REGISTRO EM PRONTUÁRIO ENSINADO PELOS DOCENTES: & $\mathrm{n}$ & $\%$ \\
\cline { 2 - 4 } Narrativa livre & 10 & 66,7 \\
Anotações pautadas na Classificação Internacional de Funcionalidade - CIF & 2 & 13,3 \\
Roteiro pré-estruturado & 2 & 13,3 \\
Registro Sequencial de Atendimento ao Paciente em Terapia Ocupacional (RSAPTO) & 1 & 6,7 \\
\hline PRINCIPAL FORMATO DO REGISTRO EM PRONTUÁRIO UTILIZADO PELOS ALUNOS NO & $\mathrm{n}$ & $\%$ \\
ESTÁGIO: & \multicolumn{2}{c}{3} \\
Narrativa livre & 9 & 27,3 \\
Roteiro pré-estruturado definido pela instituição & 7 & 21,2 \\
Roteiro pré-estruturado elaborado pelo setor de TO & 1 & 3 \\
Narrativa livre e roteiro estruturado & 2 & 6,1 \\
Anotações pautadas na Classificação Internacional de Funcionalidade - CIF & 1 & 3 \\
Não realizo evolução direta no prontuário. & & \\
\hline
\end{tabular}

Fonte: elaborado pelos próprios autores

Observou-se que outros tipos de registros, como os voltados a compra/controle de materiais, a elaboração de indicadores de produtividade e da qualidade assistencial são reduzidamente abordados no processo de ensino aprendizagem da Terapia Ocupacional em contextos hospitalares.

Gráfico 1 - Indicações dos tipos de registros abordados no processo de ensino aprendizagem da Terapia Ocupacional em Contextos Hospitalares.

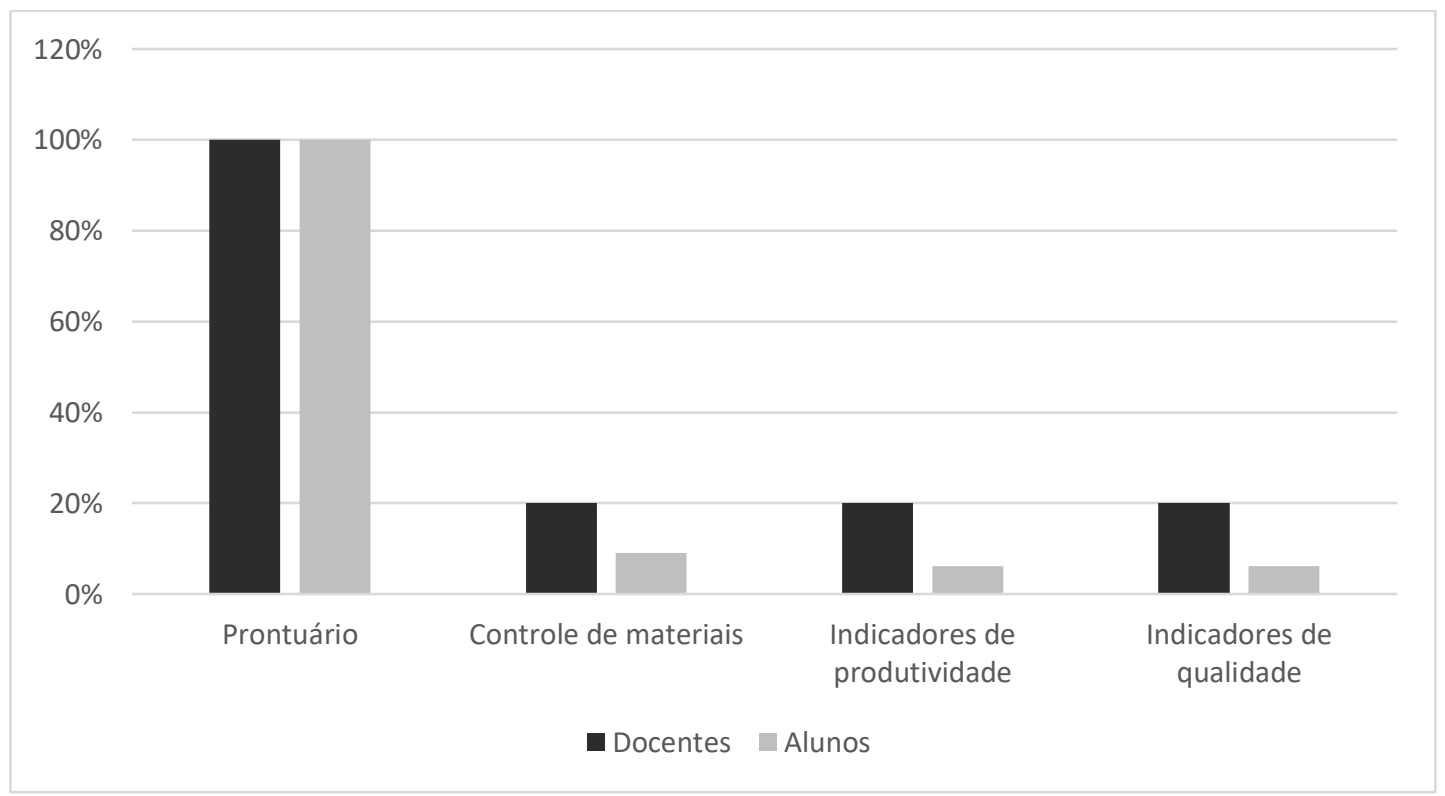

Fonte: Elaporado pelos próprios autores. 
A partir dos cruzamentos estatísticos efetivados (qui-quadrado) não foram encontradas associações com significância na categoria dos docentes. Já para a categoria dos alunos foi constatado que:

Os alunos que afirmam realizar previsão de tempo na rotina assistencial para o procedimento do registro em prontuário apresentam registros de avaliação mais completos $(\mathrm{p}<0,01)$,

Os alunos que afirmam realizar previsão de tempo na rotina assistencial para o procedimento do registro em prontuário apresentam anotações referentes as intervenções com conteúdos mais completos $(\mathrm{p}<0,01)$,

Os alunos que utilizam roteiros pré-estruturados para a elaboração dos registros apresentam conteúdos mais completos nas anotações referentes a avaliação $(p=0,03)$ e referentes as intervenções efetivadas $(p=0,04)$.

\section{DISCUSSÃO}

De modo geral, constatou-se que as informações relatadas por docentes e alunos como componentes do registro em prontuário exigidos/efetivados no processo de ensino aprendizagem mostram-se em conformidade com os apectos preconizados pelas diretrizes da classe profissional.

No entanto, é importante chamar a atenção para a necessidade da realização de pesquisas documentais que se proponham a analisar diretamente o registro terapêutico ocupacional nos prontuários dos hospitais universitários para obtenção de melhores indicadores comprobatórios da qualidade documental, visto que fragilidades relacionadas à qualidade dos registros em prontuário têm se apresentado como um problema contemporâneo em diferentes profissões (DA SILVA et al., 2016; ALVES; SPILMAN; POTON, 2015) incluindo a Terapia Ocupacional (RISCHMULLER; FRANZSEN, 2012; PELISSARI, PALHARES, 2015; BUCHANAN JELSMA; SIEGRIED, 2016). 
Em específico, como déficits vinculados ao conteúdo dos registros proferidos pelos docentes, observou-se menor frequência de orientações acerca de informações sobre prognóstico, estimativa de atendimentos e registro acadêmico (RA) do estagiário ao final da anotação. Para além de inconsistências referentes à informação sobre prognóstico, estimativa de atendimentos e RA, os alunos evidenciaram complementarmente no relato de suas práticas escassa inserção de informações sobre diagnóstico terapêutico ocupacional, plano terapêutico, contatos telefônicos e discussões interdisciplinares, assim como informações do que foi alcançado.

Tais conteúdos são preconizados pelas diretrizes da profissão e de acordo com Matsuda et al. (2006), a falta de informações preconizadas implicam na não oferta de aportes ao processo de cuidado de modo contínuo e qualificado.

A ausência do registro do plano terapêutico, bem como de informações dos resultados alcançados impossibilitam a parametrização de ganhos, fator que compromete a produção de dados que comprovem a eficácia das práticas estabelecidas.

Segundo Chaves et al., (2010), é necessário estimular a sistematização de avaliações que envolvam em sua estruturação informações sobre a coleta de dados, o estabelecimento de objetivos terapêuticos e a mensuração dos resultados. No entanto, poucas escalas de avaliação específicas de Terapia Ocupacional encontram-se validadas no Brasil, fator que pode contribuir com a dificuldade dos alunos no apontamento de dados sobre os resultados obtidos (CHAVES et al., 2010).

Ressalta-se que há uma exigência crescente no atual modelo de gestão hospitalar para a estruturação de avaliações e intervenções em bases científicas que possibilitem a visibilidade de indicadores sobre a eficácia e a eficiência do serviço prestado.

De modo simplificado, a eficiência do serviço refere-se a dados sobre a produtividade enquanto que a eficácia relaciona-se a resolutividade dos problemas. Segundo Rossaneis et al., (2015), tais indicadores têm respaldado a tomada de decisões das gerências das instituições de saúde na busca por melhorias de seus processos de trabalho e resultados. 
De acordo com Kudo (2018), a construção de indicadores dos processos assistenciais apresenta-se como um desafio para os terapeutas ocupacionais inseridos nos hospitais, visto exigências institucionais cada vez maiores para a implementação de ações e elaboração de instrumentos que possibilitem a mensuração, comparação e avaliação das ações prestadas.

A falta de indicadores claros configura-se como um problema para a prática da Terapia Ocupacional, visto contribuir para a limitação dos encaminhamentos realizados pela equipe, assim como para a produção de evidências acerca da eficácia da atuação (SKJUTAR et.al, 2010).

O fato do desenvolvimento de indicadores apresentar-se como um desafio para os terapeutas ocupacionais na prática assistencial hospitalar pode ser justificado pelo seu processo de formação. Como parte dos dados obtidos neste estudo, por exemplo, verificou-se que a formação na graduação em Terapia Ocupacional na área hospitalar apresenta lacunas na oferta de aporte para o desenvolvimento de registros de indicadores de produtividade, indicadores de qualidade e registros voltados a compras e controle de materiais, fator que não atende o desenvolvimento de competências e habilidades preconizadas nas diretrizes curriculares nacionais que expressam que os profissionais devem conhecer os principais métodos de avaliação e registro para formulação de objetivos, estratégias de intervenção e de verificação da eficácia das ações propostas em Terapia Ocupacional, bem como estar aptos a fazer o gerenciamento da força de trabalho, dos recursos físicos e materiais e de informação (BRASIL, 2002).

Frente a este cenário, considerando que o próprio mercado de trabalho acaba por exigir conhecimentos sobre gestão e planejamento em saúde é necessário que haja maiores investimentos no processo de formação dos terapeutas ocupacionais sobre tais conteúdos (SANTOS; MENTA, 2017).

A baixa frequência da descrição de informações sobre prognóstico sinalizada pelos participantes pode hipoteticamente ocorrer frente a especificidade da clientela atendida, a qual no momento da hospitalização apresenta-se clinicamente instável fator que interfere na previsão de um prognóstico ocupacional a curto prazo. Acredita-se que dificuldades vinculadas ao registro, referente a estimativa de atendimentos, também possa ocorrer atrelada a especificidade deste contexto de atuação em que o período de hospitalização e de 
alta hospitalar está condicionada a decisão médica, assim como ao fato da assistência envolver, por vezes, a finitude da vida.

Em relação ao diagnóstico terapêutico ocupacional, pode-se considerar que sua escassa inserção no registro em prontuário se dê pela ausência de discussão nacional acerca desta elaboração. Não obstante, a necessidade do aprimoramento profissional acerca do conceito de ocupação (MAGALHÃES, 2013), também pode vir a configurar-se como um fator dificultador para esta descrição.

De acordo com o artigo $1^{\circ}$ da resolução nº 81 do COFFITO, o diagnóstico terapêutico ocupacional vincula-se à avaliação cinética-ocupacional em que são consideradas alterações psico-físico-ocupacionais dos clientes (COFFITO, 1987). O eixo central do diagnóstico deve ser a ocupação, visto ser este o cerne da atuação profissional (BOMBARDA, et al., 2018). Diante de tais conceituações, o uso de classificações como a da CID-10 e DSM-V mostram-se como práticas equivocadas de registros referentes ao diagnóstico terapêutico ocupacional.

Nesta vertente, questiona-se também a pertinência do uso da Classificação Internacional de Funcionalidade - CIF como diagnóstico terapêutico ocupacional, considerando ser esta uma ferramenta de classificação utilizada por múltiplas categorias profissionais.

De acordo com a resolução 445, do COFFITO, que fixa e estabelece os parâmetros assistenciais terapêuticos ocupacionais nas diversas modalidades prestadas pelo terapeuta ocupacional, o desenvolvimento do diagnóstico terapêutico ocupacional deve ser favorecido pela realização de um processo avaliativo consistente, o qual deve envolver a identificação das:

“...habilidades e limitações do paciente para a realização das Atividades da Vida Diária, Atividades Instrumentais de Vida Diária, atividades educacionais, de trabalho, lúdicas, de lazer, descanso, sono e participação social, incluindo: fatores do cliente, tais como as estruturas e funções corporais; padrões de desempenho (hábitos, rotinas, papéis e padrões de comportamento); contextos e ambientes - cultural, físico, ambiental, social e espiritual e as demandas das atividades que afetem o desempenho ocupacional, entre outros" (COFFITO, 2014. p.129). 
Bombarda et al., (2018) afirmam que a descrição do diagnóstico terapêutico ocupacional tem se apresentado como um aspecto de grande dificuldade na prática profissional. Os autores recomendam como estratégia de facilitação para esta elaboração atenção as demandas estratificadas e correlação com o impacto destas na qualidade de vida e na participação social do paciente.

Para tanto, considera-se que o diagnóstico terapêutico ocupacional é um componente do registro que demanda investimentos atuais sobre sua configuração. Acredita-se ser necessário o desenvolvimento de estudos nesta temática que visem compreender o processo de formulação do diagnóstico exercido pelos profissionais e o raciocínio profissional atrelado a esta execução, bem como considera-se importante o fomento de discussões ampliadas que permitam maior clareza deste delineamento na área, o que contribuirá para melhor direcionamento do processo de ensino aprendizagem referente a este conteúdo.

Verificou-se que a Resolução $\mathrm{n}^{\circ} 415$ de 19 de maio de 2012 do COFFITO que normatiza a composição mínima das informações do registro em prontuário no Brasil (COFFITO, 2012) é de conhecimento dos docentes, mas que, provavelmente, os mesmos não realizam sua apresentação no contexto da formação, visto afirmação de desconhecimento referida pela maior parte dos alunos (72,7\%), o que vai ao encontro dos achados de Bombarda e Palhares (2015) que verificaram que terapeutas ocupacionais desconhecem esta resolução.

Já em relação ao formato de registro utilizado, a narrativa livre foi apontada pelos docentes e alunos como um dos principais modelos utilizados para a execução dos registros em prontuário na graduação, fator que justifica os apontamentos dos estudos de Panzeri (2013) e Bombarda e Palhares (2015) referentes ao uso da narrativa livre como principal modelo de registro utilizado pelos terapeutas ocupacionais.

Todavia, segundo Bombarda e Palhares (2015), "a narrativa livre consiste em uma escrita descritiva que demanda maior gasto de tempo na busca de informações peculiares, visto necessidade da leitura do todo para a visualização de dados específicos". Além disso, há segundo Parkinson et al., (2011) e Kielhofner et al., (2004) uma dificuldade dos profissionais em descrever com concisão suas práticas no modelo narrativo. 
Deste modo, pressupõe-se que a apresentação e ou elaboração de roteiros/modelos de registros possam vir a configurar-se como bases instrumentais importantes no processo de ensino aprendizagem por favorecer a minimização de dúvidas dos alunos sobre o que e como registrar.

Foi possível observar uma associação entre o uso de roteiros pré estruturados com a qualidade dos registros, o que significa que alunos que afirmaram conteúdos em maior conformidade com o que é preconizado pelas diretrizes da profissão foram aqueles que sinalizaram uso de algum tipo de modelo elaborado pelo setor ou pela instituição. Este dado vai ao encontro da recomendação realizada por Stumpf e Freitas (1997) aos hospitais universitários, referente à necessidade da criação de roteiros padronizados, específicos por especialidades, como estratégia para melhorar a qualidade das informações e agilizar a recuperação de dados específicos.

Diante do exposto, conclui-se que o processo de ensino aprendizagem do registro em prontuário no âmbito da graduação brasileira evidencia fragilidades, fator que denota a necessidade de maiores investimentos na qualificação da formação para a prática documental.

\section{CONSIDERAÇÕES FINAIS}

Os registros em prontuário constituem-se como uma importante ferramenta no âmbito assistencial, administrativo, educacional, jurídico e científico, fator que enfatiza a necessidade da ampliação do debate desta temática na área da Terapia Ocupacional.

A partir do entendimento que as anotações provenientes do processo de avaliação intervenção e alta são fonte de dados para pesquisas que buscam evidências sobre as melhores práticas e resultados, bem como que o registro em prontuário configura-se como uma via do discurso público profissional, a qualificação das anotações apresenta-se como uma ação que contribuirá para maior reconhecimento da profissão.

Sendo assim, o registro em prontuário é um tema contemporâneo que precisa ser ampliado na Terapia Ocupacional, tanto ao que concerne a qualificação do processo de 
formação, como em relação ao diálogo sobre as suas diretrizes pela classe profissional e à investimentos em pesquisas que se debrucem a estudar as variáveis que permeiam a prática deste procedimento.

\section{REFERÊNCIAS}

ALVES, M.A.; SZPILMAN, A.R.M.; POTON, W.L. Avaliação do registro médico nos prontuários de um ambulatório de ensino. Rev. Bras. Pesq. Saúde. Vila Velha, ES, v.17, n.3, p. 69-77, 2015.

BOMBARDA, T.B.; PALHARES, M.S. O registro de práticas interventivas da Terapia Ocupacional na educação inclusiva, Cad. Ter.Ocup.UFSCar, São Carlos, v.23, n.2, p.285-294, 2015. Disponível em: https://doi.org/10.4322/0104-4931.ctoA00496 Acesso em 3 de maio de 2019.

BOMBARDA, T.B. et al. A prática de registros em Terapia Ocupacional: reflexões sobre os fundamentos técnicolegais da resolução COFFITO-415, Revista de Terapia Ocupacional da USP, v.29, n.1, p.85-91, 2018. Disponível em https://doi.org/10.11606/issn.2238-6149.v29i1p85-91 Acesso em 3 de maio de 2019.

BRASIL. Ministério da Educação. Diretrizes Curriculares Nacionais do Curso de Graduação em Terapia Ocupacional. Resolução CNE/CES 06/2002, publicada no D.O.U 04/03/2002, Seção 1, p. 12.

BUCHANAN, H.; JELSMA, J.; SIEGRIED, N. Practice-based evidence: evaluating the quality of occupational therapy patient records as evidence for practice. South African Journal Occupational Therapy, v.46, n.1, p.65-73, 2016. Disponível em http://dx.doi.org/10.17159/2310-3833/2016/v46n1a13 Acesso em 5 de julho de 2018.

CHAVES, G.F.S. et al. Escalas de avaliação para Terapia Ocupacional no Brasil. Revista de Terapia Ocupacional da USP, v.21, n.3, p.240-246, 2010. Disponível em https://doi.org/10.11606/issn.2238-6149.v21i3p240-246 Acesso em 5 de julho de 2018.

CONSELHO FEDERAL DE FISIOTERAPIA E TERAPIA OCUPACIONAL (COFFITO). Resolução nºs1, de 9 de maio de 1987. Baixa Atos Complementares à Resolução COFFITO-8, relativa ao exercício profissional do terapeuta ocupacional, e à Resolução COFFITO-37, relativa ao registro de empresas nos Conselhos Regionais de Fisioterapia e Terapia Ocupacional, e dá outras providências. (D.O.U no․ 093, Seção I, 21/05/87). Disponível em: https://www.coffito.gov.br/nsite/?p=2839 Acesso em 17 de maio de 2019.

CONSELHO FEDERAL DE FISIOTERAPIA E TERAPIA OCUPACIONAL (COFFITO). Resolução nº $\mathbf{4 1 5}$, de 19 de maio de 2012. Dispõe sobre a obrigatoriedade do registro em prontuário pelo terapeuta ocupacional, da guarda e do seu descarte e dá outras providências. (D.O.U №99, seção 1, 23/05/2012). Disponível em: https://www.coffito.gov.br/nsite/?p=3178 Acesso em: 01 de junho de 2018.

CONSELHO FEDERAL DE FISIOTERAPIA E TERAPIA OCUPACIONAL (COFFITO). Resolução n⿳0029, de 8 de julho de 2013. Reconhece e disciplina a especialidade de Terapia Ocupacional em Contextos Hospitalares, define as áreas de atuação e as competências do terapeuta ocupacional especialista em Contextos Hospitalares e dá outras providências. (D.O.U neção 169, 1, 2/09/2013). Disponível em: http://www.atohosp.com.br/uploads/files/20170328005352.pdf Acesso em 02 de junho de 2018.

CONSELHO FEDERAL DE FISIOTERAPIA E TERAPIA OCUPACIONAL (COFFITO). Resolução n⿳0445, de 26 de abril de 2014. Altera a Resolução-COFFITO n 418/2011, que fixa e estabelece os Parâmetros Assistenciais Terapêuticos Ocupacionais nas diversas modalidades prestadas pelo Terapeuta Ocupacional.(D.O.U. nำ203, seção1, 21/10/2014). Disponível em: http://www.crefito.com.br/repository/legislacao/RESOLUCA044514.pdf Acesso em 27 de julho de 2019.

CONSELHO FEDERAL DE FISIOTERAPIA E TERAPIA OCUPACIONAL (COFFITO). Exame de conhecimento para concessão do título de especialista nas áreas de Fisioterapia e Terapia Ocupacional. Edital de abertura. $72 \mathrm{p}$, 2018. Disponível em: 
http://www.institutoexcelenciapr.com.br/sistema/uploads/EDITAL_PROVA_NACIONAL_DE_ESPECIALIDADES _-_EDICAO_2018.pdf Acesso em 25 de maio de 2019.

CONSELHO FEDERAL DE FISIOTERAPIA E TERAPIA OCUPACIONAL (COFFITO). Edital de homologação das inscrições. 115p; 2018b. Disponível em: http://www.institutoexcelenciapr.com.br/sistema/uploads/EDITAL_DE_HOMOLOGACAO_DAS_INSCRICOES.p df Acesso em: 25 de maio de 2019.

DA SILVA, T. G.; et al. Conteúdo dos registros de enfermagem em hospitais: contribuições para o desenvolvimento do processo de enfermagem. Enfermagem em Foco, v.7, n.1, p.24-27, 2016. Disponível em: http://revista.cofen.gov.br/index.php/enfermagem/article/view/679/293. Acesso em: 15 maio 2019. DOI: https://doi.org/10.21675/2357-707X.2016.v7.n1.679

DE CARLO, M.M.R.P. A especialidade profissional dos terapeutas ocupacionais em contextos hospitalares e suas áreas de atuação. In: I CONGRESSO DE TERAPIA OCUPACIONAL EM CONTEXTOS HOSPITALARES E CUIDADOS PALIATIVOS-ATOHOSP, 47., 2014, Ribeirão Preto. Anais...Revista do Hospital das Clínicas e da Faculdade de Medicina de Ribeirão Preto, 2014. p.23-24.

FANGEL, L.M.V. et al. A formação de terapeutas ocupacionais para contextos hospitalares: experiência da Universidade de Brasília. In: SILVA, R.A.S.; BIANCHI, P.C.; CALHEIROS, D.S. (orgs). Formação em Terapia Ocupacional no Brasil: pesquisas e experiências no âmbito da graduação e pós-graduação. São Paulo, FioCzar. 2018. Cap.V.p.109-121.

FEARING, V.G. Occupational therapists chart a course through the health record. Canadian Journal of Occupational Therapy, v. 60, n.5, p.232-240. 1993. Disponível em: https://doi.org/10.1177/000841749306000504 Acesso em abril de 2019.

GIL, A.C. Como elaborar projetos de pesquisa. 4ed. São Paulo: Atlas, 2002.

GIL, A.C. Métodos e técnicas de pesquisa social. 6. ed. São Paulo: Atlas, 2008.

GUIMARÃES, P.R.B. Métodos quantitativos estatísticos. Curitiba, IESD Brasil AS, 1 ed., 2008.

KIELHOFNER, G.; et al. Documenting Outcomes of Occupational Therapy: The center of outcomes research and education. American Journal of Occupational Therapy, v.58, p.15-23, 2004. Disponível em: https://doi.org/10.5014/ajot.58.1.15 Acesso em agosto de 2018.

KUDO, A.M. Gerenciamento de serviço de Terapia Ocupacional em contextos hospitalares e procedimentos no Sistema Único de Saúde. In: DE CARLO, M.M.R.P.; KUDO, A.M. Terapia Ocupacional em contextos hospitalares e cuidados paliativos. São Paulo, Editora Payá, 2018. Cap.3. p.49-78.

MAGALHÃES, L. Ocupação e atividade: tendências e tensões conceituais na literatura anglófona da terapia ocupacional e da ciência ocupacional. Cad. Ter. Ocup. UFSCar, São Carlos, v.21, n.2, p.255-263, 2013. Disponível em: http://doi.editoracubo.com.br/10.4322/cto.2013.027 Acesso em 6 de fevereiro de 2020.

MATSUDA, L.M.; et al. Anotações/registros de enfermagem: instrumento de comunicação para a qualidade do cuidado? Revista Eletrônica de Enfermagem, v.8, n.3, p.415-421, 2006. Disponível em: https://doi.org/10.5216/ree.v8i3.7080 Acesso em abril de 2019.

PANZERI, C.S.B. A prática da documentação clínica ambulatorial sob a ótica dos terapeutas ocupacionais. 2012.166f. Dissertação (Mestrado em terapia ocupacional na área de concentração: processos de intervenção em Terapia Ocupacional), UFSCar, São Carlos, 2013.

PARKINSON, S.; et al. Enhancing professional reasoning through the use of evidence-based assessment, robust case formulations and measurable goals. British Journal of Occupational Therapy, v.74, n.3, p.148- 52, 2011.

PELISSARI, D.C.; PALHARES, M.S. O registro da intervenção no prontuário pelo terapeuta ocupacional em um ambulatório infanto-juvenil. Cad.Ter.Ocup. UFSCar, São Carlos, v.23, n.4, p.711-722, 2015. 
PINHEIRO, C.A. Diferentes práticas hospitalares do terapeuta ocupacional na realidade brasileira, de norte a sul. In: I CONGRESSO DE TERAPIA OCUPACIONAL EM CONTEXTOS HOSPITALARES E CUIDADOS PALIATIVOSATOHOSP, 47., 2014, Ribeirão Preto. Anais...Revista do Hospital das Clínicas e da Faculdade de Medicina de Ribeirão Preto, 2014. p.24.

RISCHMULLER, R.; FRANZSEN, D. Assessment of record keeping at schools for learners with special educational needs in the Western Cape. South African Journal of Occupational Therapy, v.42, n.2, p.13-20, 2012.

ROSSANEIS, M.A. et al. Indicadores de qualidade da assistência: opinião de enfermeiros gerentes de hospitais de ensino. Cogitare Enfermagem, V.20, n.4, 2015. Disponível em: https://revistas.ufpr.br/cogitare/article/view/41734/26785 Acesso em: 16 maio 2019.

SANTOS, R.S.; MENTA, S.A. A formação do terapeuta ocupacional para gestão de serviços de saúde: um estudo em bases curriculares. Cad. Ter. Ocup. UFSCar, São Carlos, v. 25, n. 1, p. 43-51, 2017. Disponível em: http://dx.doi.org/10.4322/0104-4931.ctoA00710 Acesso em: 16 de maio de 2019.

SKJUTAR, A.; et al. Indicator of need for Occupational Therapy in patients whith chronic pain: occupational therapisths' focus groups. Occupational Therapy Internacional, v.17,p.93-103, 2010. Disponível em: https://doi.org/10.1002/oti.282 Acesso em 16 de maio de 2019.

STUMPF, M. K.; FREITAS, H.M.R. A gestão da informação em um hospital universitário: o processo de definição do Patient Core Record. Rev. adm. contemp., Curitiba , v. 1, n. 1, p. 71-99, Apr. 1997. Disponível em: www.scielo.br/scielo.php?script=sci_arttext\&pid=S141565551997000100005\&lng=en\&nrm=iso Acesso em $01 \mathrm{de}$ junho de 2018 .

WORLD FEDERATION OF OCCUPATIONAL THERAPY - WFOT. Minimum standards for the education of occupational therapists - $2016 . \quad$ revised $\quad$ Disponível em: http://www.wfot.org/ResourceCentre/tabid/132/did/841/Default.aspx Acesso em 16 de maio de 2019.

\section{$(\mathrm{cc}) \mathrm{Br}$}

Este trabalho está licenciado com uma Licença Creative Commons - Atribuição 4.0 Internacional. 